\title{
A theoretical investigation into quantitative modal logic
}

\author{
Churn-Jung Liau ${ }^{\mathrm{a}, *}$, Bertrand I-Peng Lin ${ }^{\mathrm{b}}$ \\ ${ }^{a}$ Institute of Information Science, Academia Sinica, Taipei, Taiwan \\ ${ }^{\mathrm{b}}$ Department of Computer Science and Information Engineering, National Taiwan University, Taipei, Taiwan
}

Received August 1993; revised November 1994

\begin{abstract}
Quantitative modal logic (QML) is a multi-modal formulation of possibilistic reasoning with the capacity of representing and reasoning about higher order uncertainty. In this paper, some results about QML and possibility theory are investigated. First, the concept of filtration in classical modal logic is introduced into QML and the fundamental filtration theorem is proved. Second, as a corollary, the finite model property of QML is provided. Finally, the theoretical and practical implications of finite model property are discussed.
\end{abstract}

Keywords: Approximate reasoning; Possibilistic logic; Quantitative modal logic; Filtration; Possibility theory

\section{Introduction}

Possibility theory [14] as a basis of uncertainty management has received more and more attention recently $[2,5]$. There are many different approaches for applying possibility theory to approximate reasoning. Among them, possibilistic logic [4] is one of the most important. Dubois and Prade successfully combine possibility theory and classical logic to create this logic and its deduction method. In $[6,10,12]$, it is shown that by formulating possibilistic reasoning as a king of modal logics, its expressive power can be enhanced to represent and reason about higher order uncertainty. Furthermore, many deduction methods for classical modal logics (such as those in [7]) can be modified to do possibilistic reasoning without too much difficulty $[11,13]$. In this paper, we would like to carry out some theoretical investigation into a modal logic for possibilistic reasoning, called quantitative modal logic(QML). First, we will introduce the concept of filtration into QML and prove its fundamental filtration theorem. Second, as a corollary, the finite model property of QML is proved. Finally, some theoretical and practical implications are discussed. In the following sections, we will present these results sequentially, after the review of possibilistic logic and QML.

\footnotetext{
*Corresponding author. E-mail: liaucj@iis.sinica.edu.tw. Tel: ( + )886-2-7883799 ext. 2417. Fax: ( + )886-2-7824814.
} 


\section{Background knowledge}

In this section, we review some necessary background knowledge for the following presentation. These are divided into three subsections. First, a brief introduction of possibility theory is given. Possibilistic logic is then introduced and a comparative review of its semantics is presented. Finally, we give a quick review of QML.

\subsection{Possibility theory}

Possibility theory is originally introduced by Zadeh [14] to model some type of uncertainty. He describes it in the context of fuzzy sets. Let $W$ be the universe of discourse, or universal set. Then a fuzzy set $A$ on $W$ can be characterized by its membership function $\mu_{A}: W \rightarrow[0,1]$. Zadeh define a possibility distribution function $\pi$ associated with $A$ as numerically equal to $\mu_{A}$, that is $\pi(w)=\mu_{A}(w)$ for all $w \in W$. However, the definition of possibility distributions can also be given in a more general context without resorting to fuzzy set theory.

Definition 1. 1. A possibility distribution on $W$ is a function $\pi: W \rightarrow[0,1]$ which satisfies the following normalized condition:

$$
\sup _{w \in W} \pi(w)=1
$$

2. The possibility measure associated with $\pi$ is a mapping $\Pi: 2^{W} \rightarrow[0,1]$ defined as

$$
\Pi(X)=\sup _{x \in X} \pi(x)
$$

for each $X \subseteq W$. Here, we assume $\sup \emptyset=0$.

On the other hand, possibility measures can be defined in an axiomatic way.

Definition 2. An axiomatic possibility measure on $W$ is a mapping $\Pi: 2^{W} \rightarrow[0,1]$ satisfying the following two axioms:

(m1) $\Pi(\emptyset)=0$ and $\Pi(W)=1$,

(m2) for each class $\Omega \subseteq 2^{W}$,

$$
\Pi(\bigcup \Omega)=\sup _{X \in \Omega} \Pi(X)
$$

It can easily be shown that these two definitions for possibility measures are equivalent. Obviously, any possibility measure associated with a possibility distribution function satisfies axioms (m1) and (m2). Conversely, given an axiomatic possibility measure $\Pi$, we can have a possibility distribution function $\pi$ defined as $\pi(x)=\Pi(\{x\})$ for each $x \in W$ and $\Pi$ is exactly the possibility measure associated with $\pi$.

Lemma $1 . \Pi$ is an axiomatic possibility measure on $W$ iff it is the possibility measure associated with a possibility distribution on $W$.

When $W$ is finite, $(\mathrm{m} 2)$ is equivalent to the following axiom

$\left(\mathrm{m} 2^{\prime}\right) \Pi(X \cup Y)=\max (\Pi(X), \Pi(Y))$ for all $X, Y \subseteq W$.

$(\mathrm{m} 1)$ and $\left(\mathrm{m} 2^{\prime}\right)$ are the usual defining axioms to connect possibility measures with consonant body of evidence [8].

The necessity measure $N$ can be defined dually as $N(X)=1-\Pi(W \backslash X)$. 


\subsection{A review of possibilistic logic}

In accordance with Zadeh's possibility theory, Dubois and Prade [4] develop a logic of partial ignorance, called possibilistic logic. The logic take propositional calculus formulas (or first order sentences) as its underlying language. Here, for convenience, we assume the underlying language is a propositional language. Let $L$ be the set of all sentences in the propositional language. Then a possibility degree on $L$ is a mapping $\Pi: L \rightarrow[0,1]$ with the restriction that $\Pi(p)=\Pi(q)$ if $p \equiv q$ is a tautology and $\Pi$ satisfies the following two axioms for all $p, q \in L$ :

(p1) $\Pi(T)=1$ and $\Pi(\perp)=0$ where $T$ and $\perp$ denote the tautology and the contradiction, respectively,

(p2) $\Pi(p \vee q)=\max (\Pi(p), \Pi(q))$.

The dual necessity degree on $L$ can be defined as $N(p)=1-\Pi(\neg p)$ for all $p \in L$. We can note the obvious correspondence between axioms $(\mathrm{m} 1),\left(\mathrm{m} 2^{\prime}\right)$ and $(\mathrm{p} 1),(\mathrm{p} 2)$.

Syntactically, the well-formed formulas (wff) of possibilistic logic, $P L_{L}$ is exactly formed by the following rule:

if $p \in L$ then $(p N c)$ and $(p \Pi c) \in P L_{L}$, where $c \in[0,1]$ is a numerical designator.

Semantically, a model $M$ for $P L_{L}$ is a triplet $\langle W, \pi, V\rangle$, where $W$ is a set of possible words, $\pi$ is a possibility distribution function on $W$ and $V: W \rightarrow(L \rightarrow\{0,1\})$ is such that each $V(w)$ is a classical truth valuation on $L$. We will write $V_{w}$ instead of $V(w)$ for each $w \in W$. For each $p \in L$, define the truth set $|p|=\left\{w \mid V_{w}(p)=1\right\}$. Given a model $M=\langle W, \pi, V\rangle, M \models(p N c)$ (resp. $\left.M \models(p \Pi c)\right)$ iff $N(|p|) \geqslant c$ (resp. $\Pi(|p|) \geqslant c)$ where $N$ and $\Pi$ is, respectively, the necessity and possibility measure associated with $\pi$. Let $S \subseteq P L_{L}$, then $M \models S$ iff for each $f \in S, M \models f$. Let $C$ be a class of models, then $S \models_{C} f$ iff for each model $M \in C$, $M \models S$ implies $M \models f$.

The semantics given above is a kind of possible world semantics [9] and has deviated somewhat from the original form given by Dubois and Prade. In fact, in [3], the principle of minimum specificity is used to define a canonical model $M^{*}$ for a set of necessity-valued wffs in $P L_{L}$. Given a set $S=\left\{\left(p_{i} N c_{i}\right) \mid 1 \leqslant i \leqslant n\right\}$, the canonical model $M_{i}^{*}$ for $\left(p_{i} N c_{i}\right)$ is a pair $\left\langle W, \pi_{i}\right\rangle$, where $W$ is the set of all truth assignments on $L$ and $\pi_{i}: W \rightarrow[0,1]$ is defined as

$$
\pi_{i}(w)= \begin{cases}1 & \text { if } w\left(p_{i}\right)=1 \\ 1-c_{i} & \text { if } w\left(p_{i}\right)=0\end{cases}
$$

The canonical model for $S$ is then $M^{*}=\left\langle W, \pi^{*}\right\rangle$, where $\pi^{*}$ is defined as $\pi^{*}(w)=\min _{1 \leqslant i \leqslant n} \pi_{i}(w)$ for each $w \in W$.

There are two main differences between the possible world semantics and canonical model semantics of possibilistic logic. The first one is that in canonical model, the truth valuation $V_{w}$ is identified with $w$. This can be achieved in possible world models when $V$ is an 1-1 and onto mapping, i.e., $V_{u}=V_{w}$ only if $u=w$ and for each truth valuation on $L$, there is a $w \in W$ such that $V_{w}$ is that valuation. We call such possible world models reduced models. It can be shown that reduced model semantics is equivalent to possible world semantics for possibilistic logic by using the filtration technique introduced in the next section, so the first difference is irrelevant. Thus, we can write a reduced model as a pair $\langle W, \pi\rangle$, and obviously, canonical models are a kind of reduced models when its possibility distribution $\pi^{*}$ is normalized. However, to what degree can a canonical model represent the class of all reduced models? When $S$ is a set of necessity-valued wffs as defined above, the canonical model of $S$ can completely characterize all its reduced models. In other words, it can easily be verified that $\langle W, \pi\rangle$ is a reduced model of $S$ iff $\pi(w) \leqslant \pi^{*}(w)$ for all $w \in W$ where $\left\langle W, \pi^{*}\right\rangle$ is the canonical model of $S .{ }^{1}$ However, when possibility-valued wffs are included in $S$, it is unclear whether there is

\footnotetext{
${ }^{1}$ This must subject to the condition that $\pi^{*}$ is normalized, i.e., $S$ is consistent.
} 
a canonical model that can characterize all reduced models of $S$ (cf. [3]). This is the second difference between possible world models (or reduced models) and canonical models. From the difference, it can be seen that possible world semantics is more flexible than canonical semantics. It is more easily adapted to the more complex cases when higher order uncertainty is represented. This is just what will be described in Section 2.3.

\subsection{Quantitative modal logic}

Although possibilistic logic provide a useful tool to model and represent uncertainty, it is inadequate in some cases. For example, if from some information source, we get a rule "Smoking implies the possibility of cancer being at least 0.8 ", but the certainty (necessity) of the rule is at most 0.7 due to the reliability of the information source, then we would like to represent this fact as $N((p \supset(\Pi(q) \geqslant 0.8))) \leqslant 0.7$, where $p$ and $q$ denote "smoking" and "getting cancer", respectively. However, it is obvious that the formula is outside the scope of possibilistic logic. This is the so-called higher order uncertainty formula. We can represent it by extending the syntax of possibilistic logic and formulating it as a kind of modal logics. QML is an attempt towards the direction. It is an extension of propositional logic with two classes of quantitative modal operators: $\langle c\rangle$ and $\langle c\rangle^{+}$for all $c \in[0,1]$.

Let $A t$ be the set of atomic formulas for the underlying propositional language. Then the wffs of QML, $Q F$ is the smallest set containing $A t$ and closed under the following conditions:

- if $f$ and $g \in Q F$, then $f \wedge g$ and $f \vee g \in Q F$,

- if $f \in Q F$, then $\neg f,\langle c\rangle f$ and $\langle c\rangle^{+} f \in Q F$, for all $c \in[0,1]$.

Another two classes of modal operators $[c]$ and $[c]^{+}$are defined as abbreviations of $\neg\langle 1-c\rangle^{+} \neg$ and $\neg\langle 1-c\rangle \neg$, respectively. Other classical connectives are defined as usual. We use lower case letters (sometimes with indices) $p, q, r$ to denote atomic formulas and $f, g, h$ to denote wffs.

A model $M$ of QML is still a triplet $\langle W, \pi, V\rangle$ where $\pi: W \rightarrow(W \rightarrow[0,1])$ is now a set of possibility distributions indexed by $W$ and each $V_{w}: A t \rightarrow\{0,1\}$ is now a truth valuation on the atomic set. Just as $V_{w}$, we also write $\pi_{w}$ instead of $\pi(w)$. Each truth valuation $V_{w}$ can be extended to the whole set $Q F$ according to the following rules:

1. $V_{w}(\neg f)=1-V_{w}(f)$,

2. $V_{w}(f \vee g)=\max \left(V_{w}(f), V_{w}(g)\right)$,

3. $V_{w}(f \wedge g)=\min \left(V_{w}(f), V_{w}(g)\right)$,

4. $V_{w}(\langle c\rangle f)=1$ iff $\Pi_{w}\left(\left\{u \mid V_{u}(f)=1\right\}\right) \geqslant c$,

5. $V_{w}\left(\langle c\rangle^{+} f\right)=1$ iff $\Pi_{w}\left(\left\{u \mid V_{u}(f)=1\right\}\right)>c$,

where $\Pi_{w}$ is the possibility measure associated with $\pi_{w}$. We write $w \models_{M} f$ if $V_{w}(f)=1$ and $M \models f$ if $V_{w}(f)=1$ for all $w \in W$. The definitions of $M \models S$ and $S \models_{c} f$ are the same as those in possibilistic logic.

\section{Filtration technique and finite model property}

As an illustrative example, let us prove that reduced model semantics are equivalent to possible world semantics for possibilistic logic in the following sense. Two models $M, M^{\prime}$ of $P L_{L}$ are said to be equivalent if for each $f \in P L_{L}, M \models f$ iff $M^{\prime} \models f$.

Theorem 1. Let $M$ be a possible world model for $P L_{L}$, then we can find an equivalent reduced model $M^{\prime}$.

Proof. The proof is carried out in two steps. Suppose $M=\langle W, \pi, V\rangle$. First, if $V$ is not onto, then for each truth valuation in $L \rightarrow\{0,1\}$ that is not in the image of $V$, introduce a new $w$ into $W$ and extend $V$ such that $V_{w}$ is that valuation. Let $W^{\prime \prime}$ be the union of $W$ and all new-introduced worlds, $V^{\prime \prime}$ be the extended $V$, and $\pi^{\prime \prime}: W^{\prime \prime} \rightarrow[0,1]$ is defined as $\pi^{\prime \prime}(w)=\pi(w)$ if $w \in W$ and 0 otherwise. Then it is obvious $M$ and $\left\langle W^{\prime \prime}, \pi^{\prime \prime}, V^{\prime \prime}\right\rangle$ 
are equivalent. Second, define a equivalence relation $\simeq$ on $W^{\prime \prime}$ such that $w \simeq u$ iff $\left.V_{w}^{\prime \prime}=V_{u}^{\prime \prime}\right)$. Let $[w]$ denote the $\simeq$-equivalence class containing $w$ and $W^{\prime}=\left\{[w] \mid w \in W^{\prime \prime}\right\}$. Define $\pi^{\prime}$ and $V^{\prime}$ such that $\pi^{\prime}([w])=$ $\sup _{u \in[w]} \pi^{\prime \prime}(u)$ and $V_{[w]}^{\prime}=V_{w}^{\prime \prime}$. Then for each $f \in P L_{L}, \Pi^{\prime}(|f|)=\Pi^{\prime \prime}(|f|)$, so $M^{\prime}=\left\langle W^{\prime}, \pi^{\prime}, V^{\prime}\right\rangle$ is equivalent with $M^{\prime \prime}$ and is the required reduced model.

When the underlying propositional language is finite, the reduced models must have finite possible worlds. Thus we have the finite model property of $P L_{L}$ as a corollary.

Corollary 1. If $S \subseteq P L_{L}$ and there is a model $M$ such that $M \models S$, then there is a finite model $M^{\prime}$ such that $M^{\prime} \vDash S$.

The corollary is by no means a new result because the canonical semantics has provided a constructive approach to prove it, at least in the case of $S$ being a set of necessity-valued wffs. However, the technique used here is more easily adapted to QML.

As we have seen in the preceding section, the applicability of canonical model semantics based on the principle of minimum specificity is limited. It is unknown what the canonical model for $P L_{L}$ should be when possibility-valued wffs are included. It is even unknown whether there exists such model for general case. Due to the complexity of QML semantics (each world is associated with a possibility distribution), it is even more unlikely to have a minimum specific model for a given set of wffs in $Q F$. This somewhat precludes the use of canonical semantics as a constructive approach to prove finite model property. Another type of canonical model method, which take the maximal consistent sets of wffs as the possible worlds, is of course plausible [10]. However, that method is far complicated than the filtration technique. Furthermore, filtration technique facilitates us to construct an equivalent (in some sense, of course) finite model from any model of QML, not just a canonical one.

By observing the proof of Theorem 1 more carefully, we find that the result depends on the partition of $W^{\prime \prime}$ according to some equivalence relation. This is a kind of filtration technique. Here the equivalence relation is defined such that two worlds are equivalent if they have same truth valuations on $L$, so if $L$ is finite, the equivalence classes are finite. Thus the finite model property holds whether $S$ is finite or not. We can do so because the characteristic attribute of a world in possibilistic logic model is its associated truth valuation. However, for QML models, because we associated a world with a possibility distribution in addition to a truth valuation, even though two worlds have the same classical truth valuations, they may have very different associated possibility distributions. Because the number of possibility distributions is potentially infinite, there may be some set of consistent wffs which has no finite models.

Example 1. Consider the wff $f_{i}=\left([1 / i] p \wedge \neg[1 / i]^{+} p\right)$ where $p \in A t$ is an atomic propositional symbol and let $S=\left\{\langle 1\rangle f_{i} \mid i \in Z^{+}\right\}$, then $S$ have no finite model. The argument is as follows. Assume $M=\langle W, \pi, V\rangle$ is a model of $S$, i.e., $M \models S$ and $W$ is finite, then pick up any world $w \in W$, we have $V_{w}\left(\langle 1\rangle f_{i}\right)=1$ for all $i \in Z^{+}$. Since $W$ is finite, this means there exists $w_{i} \in W$ such that $\pi_{w}\left(w_{i}\right)=1$ and $V_{w_{i}}\left(f_{i}\right)=1$ for each $i$. Once again, since $W$ is finite, there must exist different $i, j \in Z^{+}$such that $w_{i}=w_{j}=w^{\prime}$ for some $w^{\prime}$. Then $V_{w^{\prime}}\left(f_{i}\right)=V_{w^{\prime}}\left(f_{j}\right)=1$ for some $i \neq j$, but it is impossible since $V_{w^{\prime}}\left(f_{i}\right)=1$ only when $N_{w^{\prime}}(p)=1 / i$.

This example shows that finite model property does not hold for QML when $S$ is infinite even though the underlying propositional language contains only finite atomic symbols. However, the filtration technique used in Theorem 1 can be applied to QML if we modify the definition of the equivalence relation appropriately. From now on, we assume that $\Gamma \subseteq Q F$ is a set of wffs closed under subformulas, that is, if $f \in \Gamma$ then any subformulas of $f$ is also in $\Gamma$. Given a QML model $M=\langle W, \pi, V\rangle$, define the equivalence relation $\simeq_{\Gamma}$ on $W$ as

$$
u \simeq_{\Gamma} w \Leftrightarrow V_{u}(f)=V_{w}(f) \quad \forall f \in \Gamma .
$$


The notations $[w]$ is same as above. Let $[X]=\{[w] \mid w \in X\}$ for all $X \subseteq W$. Define a representative selection function as $r:[W] \rightarrow W$ such that $r([w]) \in[w]$ for all $w \in W$. The function arbitrarily select a representative from each equivalence class. The possibility distribution of $r([w])$ will be taken as that of $[w]$. Define $\pi^{r}:[W] \rightarrow([W] \rightarrow[0,1])$ as

$$
\pi_{[w]}^{r}([u])=\sup _{x \in[u]} \pi_{r([w])}(x)
$$

for all $u, w \in W$, and $V^{r}:[W] \rightarrow(A t \rightarrow\{0,1\})$ such that $V_{[w]}^{r}=V_{r([w])}$. Then the model $M^{r}=\left\langle[W], \pi^{r}, V^{r}\right\rangle$ is called the $\Gamma$-filtration of $M$ under $r$. By these definitions, we can now state the main theorem of the paper.

Theorem 2 (Fundamental filtration theorem). If $M^{r}$ is the $\Gamma$-filtration of $M$ under $r$, then for all $f \in \Gamma$ and $w \in W, V_{w}(f)=V_{[w]}^{r}(f)$.

Proof. The proof is by induction on the structure of $f$. For the sake of brevity, assume $r([w])=y$. Note that $w \simeq_{\Gamma} y$ by definition.

Basis: If $f=p \in A t$, then by definition of $V^{r}$,

$$
V_{[w]}^{r}(p)=V_{y}(p)=V_{w}(p) \text { since } p \in \Gamma \text {. }
$$

Step: The interesting cases are when $f=\langle c\rangle g$ or $f=\langle c\rangle^{+} g$. Assume $|g|$ is the truth set of $g$ in the model $M$, then by induction hypothesis, the truth set of $g$ in the filtration $M^{r}$ is $[|g|]$, and we have the following equations:

$$
\Pi_{w}(|g|)=\sup _{x \in|g|} \pi_{w}(x)
$$

and

$$
\begin{aligned}
\Pi_{[w]}([|g|]) & =\sup _{[u] \in[|g|]} \pi_{[w]}^{r}([u]) \\
& =\sup _{[u] \in[|g|]} \sup _{x \in[u]} \pi_{y}(x) \\
& =\sup _{x \in|g|} \pi_{y}(x) \\
& =\Pi_{y}(|g|) .
\end{aligned}
$$

Since $w \simeq_{\Gamma} y$ and $f=\langle c\rangle g$ or $\langle c\rangle^{+} g \in \Gamma$, we have $\Pi_{w}(|g|) \geqslant c$ (resp. $>c$ ) iff $\Pi_{y}(|g|) \geqslant c$ (resp. $\left.>c\right)$. Combining this with the equations above, we have the required result.

Finite model property of QML for finite set of wffs follows as a corollary of the theorem. Note $M^{r}$ is finite when $\Gamma$ is finite. Let $S u b(S)$ denote the closure set of $S$ under subformula.

Corollary 2. For each $f \in Q F, f$ has a model iff it has a finite model.

Proof. Let $\Gamma=S u b(\{f\})$ and $M$ be a model of $f$, then the $\Gamma$-filtration of $M$ under any $r$ can serve the purpose.

Let $\boldsymbol{D}$ and $\boldsymbol{F D}$ denote the class of all models and the class of all finite models, respectively. 
Corollary 3. For all finite $S \subseteq Q F$ and $f \in Q F, S \models_{D} f$ iff $S \models_{F D} f$.

Proof. Let $\Gamma=S u b(S \cup\{\neg f\})$.

\section{Implications of the result}

\subsection{The theoretical implication}

From a viewpoint of possibility theory, the models for QML defined above are a kind of distribution based model because we associated a possibility distribution with each world. For comparison, we call these models $\mathrm{d}$-models. We can also define the models in a measure base. Define an m-model of QML as a triplet $\langle W, \Pi, V\rangle$, where $W$ and $V$ are the same as that in d-models and $\Pi: W \rightarrow\left(2^{W} \rightarrow[0,1]\right)$ is a set of possibility measures such that $\Pi_{w}$ satisfies axioms $(\mathrm{m} 1)$ and $(\mathrm{m} 2)$ for each $w \in W$. The reader familiar with classical modal logic can see that a-models in fact generalize the notion of the so-called minimal models or neighborhood models [1]. Let $\boldsymbol{A}$ and $\boldsymbol{F A}$ denote the class of all a-models and finite a-models, respectively. Then the following theorem follows directly from Lemma 1.

Theorem 3. For all $S \subseteq Q F$ and $f \in Q F$, we have

1. $S \models_{D}$ f iff $S \models_{A} f$, and

2. $S \models_{F D} f$ iff $S \models_{F A} f$.

A theoretical implication of the theorem is that the equivalence between standard and minimal semantics of classical modal logics is just corresponding to the equivalence between possibility distributions and axiomatic possibility measures in possibility theory. One advantage of minimal semantics is that it reflect the syntactic axioms in the semantic structure directly. However, the axiom (m2) somewhat destroys the advantage because the possibly infinite union does not have any corresponding operation in our language since QML is a finitary language. On the other hand, the axioms $(\mathrm{ml})$ and $\left(\mathrm{m} 2^{\prime}\right)$ correspond exactly to the possibilistic logic axioms (p1) and (p2). We have also seen that when $W$ is finite, $(\mathrm{m} 2)$ is reduce to $\left(\mathrm{m} 2^{\prime}\right)$. The finite a-models indeed reflect the original possibilistic logic axioms in their structures precisely. The finite model property and the preceding theorem bridge all the above-mentioned classes of models.

Corollary 4. $S \models_{A} f$ iff $S \models_{F A}$ ffor all finite $S \subseteq Q F$ and $f \in Q F$.

Thus, when we are only interested in the consequence relation between finite wffs, we can present the semantics of QML as the measure based semantics reflecting precisely the possibilistic logic axioms, irrespective of the cardinality of the underlying propositional language.

\subsection{The practical implication}

The mechanical proof methods for possibilistic logic is proposed in [3], where resolution method is used. However, the set of wffs must be converted into clausal form before resolution can be performed and this is sometimes not easy, in particular when possibility based wffs are included.

In $[12,13]$, some non-clausal proof methods, including tableau method and Gentzen sequent calculus, are proposed for QML. The discussion of full detail about these methods is beyond the scope of the paper, however, we will show that the finite model property is important on the soundness of a key rule in tableau method. 
In tableau method, a wff $f$ to be proved is negated first. Then, different rules are applied to the negated wff $\neg f$ by trying to find a model $M$ and a possible world $w$ such that $w \models_{M} \neg f$. If it can be shown that this is impossible, then the original $f$ is valid. In the course of applying the different rules, $\neg f$ is split into some subsets of wffs. Each subset is called a branch. If a branch contains an atomic wff and its negation, then the branch is closed. If all branches are closed, then the results are proved.

A key rule for this method is the so-called $\pi$-rule. Roughly, when a wff $\langle c\rangle g$ appears in a branch, then if there is a model $M$ and $w$ such that $w=_{M} f$ for all $f$ in that branch, we can find another finite model $M^{\prime}$ and $w^{\prime}$ has the same property by finite model theorem. For finite model, $w^{\prime} \models_{M^{\prime}}\langle c\rangle g$ means there exists some $u$ such that $u \models_{M^{\prime}} g$ and $\pi_{w^{\prime}}(u) \geqslant c$. Thus, for all wffs $f$ such that $\neg\langle c\rangle \neg f$ in the original branch, $f$ must be also true in $u$. So $g$, together with all $f$ 's such that $\neg\langle c\rangle \neg f$ is in the original branch, forms a new branch replacing the original branch. Note the soundness of the rule depends heavily on the finite model property because $w \models_{M}\langle c\rangle g$ does not necessarily imply the existence of $u$ satisfying $u \models_{M} g$ and $\pi_{w}(u) \geqslant c$ when $M$ is infinite.

Therefore, a practical implication of the finite model property is that the soundness of tableau method for QML can be constructed not resorting to the axiomatic system as in [13].

\section{Concluding summary}

This short note describes some theoretical work on quantitative modal logic. To accommodate the representation of higher order uncertainty, the semantics for QML is substantially more complex than that for possibilistic logic. Hence though the canonical models based on minimum specificity principle provide a neat characterization of all models for a set of wffs in possibilistic logic so that given a consistent set of necessity-valued wffs, a finite model can easily be constructed, the principle can not be adapted to QML easily. While the finite model property of possibilistic logic holds even for an infinite set of wffs, it is not the case for QML. Thus, unlike in possibilistic logic, there is not an easy approach to construct a finite model from a given set of consistent QML wffs. However, there are some methods to cope with the difficulty for proving finite model property. One of them is based on the so-called Lindeanbaum construction $[1,10]$. But the method is quite complicated. Here we use the filtration technique to achieve the same purpose. The technique provide a simple method for proof of finite model property.

The theoretical implication of the result is that a measure based semantics precisely reflecting the possibilistic logic axioms can be provided for QML and the practical implication is to provide a direct soundness verification of the rules used in the tableau methods for QML.

\section{References}

[1] B.F. Chellas, Modal Logic: An Introduction (Cambridge University Press, Cambridge, 1980).

[2] D. Dubois, J. Lang and H. Prade, Fuzzy sets in approximate reasoning. Part 2: Logical approaches, Fuzzy Sets and Systems 40 (1991) 203-244.

[3] D. Dubois, J. Lang and H. Prade, Advances in automated reasoning using possibilistic logic, in: A. Kandel, Ed., Fuzzy Expert Systems (CRC Press, Boca Raton, FL, 1992) 125-134.

[4] D. Dubois and H. Prade, An introduction to possibilistic and fuzzy logics, in: P. Smets et al., Eds., Non-Standard Logics for Automated Reasoning (Academic Press, London, 1988) 287-325.

[5] D. Dubois and H. Prade, Fuzzy sets in approximate reasoning. Part 1: Inference with possibility distributions, Fuzzy Sets and Systems 40 (1991) 143-202.

[6] L. Farinas del Cerro and A. Herzig, A modal analysis of possibility theory, in: R. Kruse and P. Siegel, Eds., Proc. of ECSQAU, Lecture Notes in Artificial Intelligence, Vol. 548 (Springer, Berlin, 1991) 58-62.

[7] M.C. Fitting, Proof Methods for Modal and Intuitionistic Logics (D. Reidel, Dordrecht, 1983).

[8] G.J. Klir and T.A. Folger, Fuzzy Sets, Uncertainty, and Information (Prentice-Hall, London, 1988).

[9] S.A. Kripke, Semantical considerations on modal logic, Acta Philosophica Fennica 16 (1963) 83-94. 
[10] C.J. Liau and I.P. Lin, Quantitative modal logic and possibilistic reasoning, in: B. Neumann, Ed., Proc. of ECAI 92 (Wiley, New York, 1992) 43-47.

[11] C.J. Liau and I.P. Lin, Reasoning about higher order uncertainty in possibilistic logic, in: J. Komorowski and Z.W. Raś, Eds., Methodologies for Intelligent Systems, Lecture Notes in Artificial Intelligence, Vol. 689, (Springer, Berlin, 1993) 316-325.

[12] C.J. Liau and I.P. Lin, Proof methods for reasoning about possibility and necessity, Internat. J. Approx. Reason. 9(4) (1993) 327-364.

[13] C.J. Liau and I.P. Lin, Gentzen sequent calculus for possibilistic reasoning, in: M. Masuch and L. Pólos, Eds., Knowledge Representation and Reasoning under Uncertainty, Lecture Notes in Artificial Intelligence, Vol. 808 (Springer, Berlin, 1994).

[14] L.A. Zade, Fuzzy sets as a basis for a theory of possibility, Fuzzy Sets and Systems 1 (1978) 3-28. 